\title{
BMJ Open Diagnostic performance of the Chronic Kidney Disease Epidemiology Collaboration (CKD-EPI) equation at estimating glomerular filtration rate in adults with diabetes mellitus: a systematic review and meta- analysis protocol
}

\author{
Neda Zafari, ${ }^{1}$ Leonid Churilov, ${ }^{2}$ Richard J Maclsaac, ${ }^{3}$ Niloufar Torkamani, ${ }^{1}$ \\ Helen Baxter, ${ }^{4}$ Katerina V Kiburg, ${ }^{3}$ Elif Ekinci ${ }^{1}$
}

To cite: Zafari N, Churilov L, Maclsaac RJ, et al. Diagnostic performance of the Chronic Kidney Disease Epidemiology Collaboration (CKD-EPI) equation at estimating glomerular filtration rate in adults with diabetes mellitus: a systematic review and metaanalysis protocol. BMJ Open 2019;9:e031558. doi:10.1136/ bmjopen-2019-031558

- Prepublication history and additional material for this paper are available online. To view these files, please visit the journal online (http://dx.doi org/10.1136/bmjopen-2019031558).

Received 10 May 2019 Revised 15 July 2019 Accepted 18 July 2019

Check for updates

(C) Author(s) (or their employer(s)) 2019. Re-use permitted under CC BY-NC. No commercial re-use. See rights and permissions. Published by BMJ.

For numbered affiliations see end of article.

Correspondence to

Dr Neda Zafari;

zafarin@student.unimelb.edu.au

\section{ABSTRACT}

Introduction Timely detection leading to the implementation of reno-protective measures reduces the progression of diabetic kidney disease. Estimated glomerular filtration rate (eGFR) is a major surrogate of kidney function. The Chronic Kidney Disease Epidemiology Collaboration (CKD-EPI) Equation is a tool to estimate GFR. This protocol outlines a systematic-review, assessing the diagnostic accuracy of the CKD-EPI equation in adults with diabetes.

Methods and analysis MEDLINE, Embase, Cochrane Central Register of Controlled Trials and grey literature will be searched for publications in English, Farsi, Dutch and Chinese from 2009 (when CKD-EPI was first introduced) to January 2019. Bridging searches will be conducted to capture literature published from January 2019 until final review publication. The inclusion criteria will be (1) study participants with diabetes; (2) age $\geq 18$ years; (3) creatinine-based CKD-EPI eGFR as index test; (4) measured GFR using the clearance/plasma disappearance of inulin, iohexol, iothalamate, diethylenetriaminepentaacetic acid (DTPA) or chromium labelled ethylenediaminetetraacetic acid (Cr-EDTA) as reference test; (5) report of the diagnostic accuracy of the index test. Exclusion criteria will be participants with renal transplant, chronic use of corticosteroids, chronic inflammatory diseases, pregnancy, non-diabetes related kidney disease, thalassaemia, heart failure, pregnancy and potential kidney donors as well as critically ill patients. Screening, eligibility check, risk of bias assessment and data extraction will be carried out by two independent reviewers. Any discrepancies will be discussed, and third-party opinion will be sought. The risk of bias will be assessed using the Quality Assessment of Diagnostic Accuracy Studies-2 tool. A quantitative synthesis of the aggregated-data will be used if the included studies are homogenous.

Ethics and dissemination No ethics approval is required. The outcome will be published in a peer-reviewed journal. The results will help researchers and clinicians evaluate
Strengths and limitations of this study

To the best of our knowledge, this will be the first systematic review to evaluate accuracy, precision and bias of the Chronic Kidney Disease Epidemiology Collaboration estimated glomerular filtration rate (eGFR) equation in people with diabetes.

- The frequency of utilising other statistical methods like interclass correlation coefficient which are more accurate in evaluating diagnostic accuracy of tests will be assessed.

- Methods of measuring GFR in different studies varies and this might affect the homogeneity of the data and limit the possibility of conducting quantitative analysis of the results.

- The definition of bias and precision could vary in different studies, hence, there may be limited scope to conduct a meta-analysis of the collected data.

the diagnostic accuracy of the creatinine-based CKD-EPI eGFR in adults with diabetes.

PROSPERO registration number CRD42018108776

\section{INTRODUCTION}

Target condition being diagnosed

Diabetic kidney disease, defined as having albuminuria or estimated glomerular filtration rate $(\mathrm{GFR})<60 \mathrm{~mL} / \mathrm{min} / 1.73 \mathrm{~m}^{2}$, affects approximately $40 \%$ of people with diabetes and is the most common cause of dialysis or renal transplantation worldwide. ${ }^{1-4}$ Moreover, having diabetic kidney disease is associated with an increased risk of cardiovascular as well as all-cause mortality. ${ }^{56}$ The development of irreversible chronic kidney disease (CKD) is characterised by a preceding phase of renal 
function decline in both type 1 and type 2 diabetes which is clinically silent, but potentially reversible. In some cases, there is a marked hyperfiltration phase before the reduction in kidney function begins. However, many people who are passing through this phase are undiagnosed due to underperformance of the current routine kidney function estimation tools. ${ }^{7-9}$ Research shows that if diagnosed early, interventions could be implemented to halt the progression of kidney function decline, potentially resulting in an improved quality of life..$^{10-12}$

\section{Reference test(s)}

GFR is considered the best overall index of normal and diseased kidney function. GFR can be measured using the clearance/plasma disappearance of exogenous filtration markers like inulin or radiotracer molecules such as technetium-99m diethylenetriamine-pentaacetic acid $\left({ }^{99} \mathrm{mTc}\right.$-DTPA $),{ }^{125}$ I-iothalamate, or ${ }^{51} \mathrm{Cr}$-EDTA which are neither secreted nor absorbed in the glomeruli. The methods used to assess measured GFR (mGFR) are expensive and require multiple serum sample collections from patients over approximately 4 hours. Hence mGFR is not considered to be a desirable screening test.

\section{Index test(s)}

Estimating GFR with endogenous filtration markers like creatinine has been proposed as a feasible and less invasive methods for evaluating kidney function. ${ }^{13}{ }^{14}$ The Modification of Diet in Renal Disease (MDRD) Study equation has gained widespread acceptance. The estimated GFR (eGFR) using this equation is reported by most clinical laboratories when measurement of serum creatinine is ordered. ${ }^{15}$ The MDRD Study equation was developed in people with CKD, and as such its major limitations are imprecision and systematic underestimation of mGFR (bias) at higher levels of renal function. ${ }^{16}$ In an attempt to overcome some of the limitations of the MDRD equation, the Chronic Kidney Disease Epidemiology Collaboration (CKD-EPI) equation was developed. ${ }^{17}$ In particular, it has been reported to reduce bias compared with the MDRD equation for GFR $>60 \mathrm{~mL} / \mathrm{min} / 1.73 \mathrm{~m}^{2}$ in various study populations. ${ }^{18} 19$ However, the diagnostic performance of the CKD-EPI equation per se in people with diabetes, especially those with eGFR above 60 or at the hyperfiltrating stage has been an issue of controversy. While some studies demonstrated a high bias and low precision and accuracy for the current equation, ${ }^{20-23}$ other studies argued that the equation's diagnostic performance is adequate in people with diabetes. ${ }^{24}{ }^{25}$ Some studies have shown occurrence of a considerable misclassification of CKD stage when using the CKD-EPI equation in people with diabetes while others found a low misclassification rate. ${ }^{2326}$ These controversial results and conclusion with regards to whether the creatinine-based CKD-EPI equation is appropriate to be used in people with diabetes, necessitates a systematic review of the current literature to possibly reach a consensus.

\section{Objectives}

We aim to systematically review the diagnostic performance of the creatinine-based CKD-EPI equation in people with diabetes looking at the equation's accuracy, precision and bias, compared with the gold standard tests of measuring GFR, in depicting (1) the kidney function at a single time point and (2) change in GFR during time. The results of this systematic review might help in reaching a consensus regarding the diagnostic performance of the creatinine-based CKD-EPI equation in people with diabetes and taking a further step to modify this equation in this specific population if indicated.

\section{METHODS AND ANALYSIS \\ Eligibility criteria}

The systematic review intends to evaluate the diagnostic performance of CKD-EPI creatinine-based eGFR formula in estimating (1) GFR at a specific timepoint or (2) GFR change during time in adults with type 1 or type 2 diabetes. Studies in English, Farsi, Dutch and Chinese since 2009 (when the CKD-EPI equation was introduced) will be obtained using electronic databases, trial registers and grey literature. The inclusion criteria are defined as: (1) study participants with type 1 or type 2 diabetes, (2) study participants aged $\geq 18$, (3) using creatinine based CKD-EPI GFR estimation equation as the index test, (4) mGFR using the clearance/plasma disappearance of inulin, iohexol, ${ }^{125}$ I-iothalamate, ${ }^{99} \mathrm{mTc}$-DTPA or ${ }^{51} \mathrm{Cr}$ EDTA as the reference test and (5) report of at least one of the diagnostic accuracy specifiers (ie, bias, accuracy and precision) of the creatinine-based CKD-EPI. Studies conducted on participants with renal transplant, chronic use of corticosteroids, chronic inflammatory diseases and non-diabetes related kidney disease, thalassaemia, heart failure, pregnancy and potential kidney donors as well as critically ill patients will be excluded.

\section{Information sources}

We will search MEDLINE (Ovid interface, 2009 onwards), Embase (Ovid interface, 2009 onwards) and the Cochrane Central Register of Controlled Trials (CENTRAL) (Wiley interface, 2009 onwards). Our search strategy will be developed using Medical Subject Headings (MeSH) and text words related to diabetes mellitus, CKD, eGFR using CKD-EPI Equation and mGFR. The electronic database search will be supplemented by searching the grey literature via trial registries, Google Advanced Search and by contacting authors where insufficient data are available. The literature search will not be limited by language; however, a date limit of 2009 onwards will be applied as this is when the CKD-EPI equation was developed. To ensure literature saturation, we will scan the reference lists of included studies or relevant reviews identified.

\section{Search strategy}

MEDLINE, Embase and CENTRAL will be searched from 2009 to January 2019 with no language limits applied. 
The specific search strategies will be created by a Clinical Librarian with expertise in systematic review searching. The MEDLINE strategy will be developed with input from the review team, then peer reviewed by a second librarian, not otherwise associated with the review, using the PRESS standard. A draft MEDLINE search strategy is included below. After the MEDLINE strategy is finalised, it will be adapted to the syntax and subject headings of the other databases. As well, the International Clinical Trials Registry Platform Search Portal and ClinicalTrials. gov will be searched for ongoing or recently completed trials, and PROSPERO will be searched for ongoing or recently completed systematic reviews. As relevant studies are identified, reviewers will check for additional relevant cited and citing articles. A bridging search will be conducted prior to publication of the review to ensure inclusion of any relevant studies published since January 2019.

Draft MEDLINE search: Ovid interface

1. Diabetes Mellitus, Type 1/or Diabetes Mellitus, Type 2/or Diabetes Mellitus/or Diabetic Nephropathies/or Diabetes Complications/or (chronic kidney disease or CKD or diabetic or diabetes or T2DM).mp.

2. (eGFR or estimating equation* or CKD-EPI or Chronic Kidney Disease Epidemiology Collaboration or cystatin* or (estimat* and (GFR or glomerular filtration))). mp.

3. (mGFR or (measure* adj5 (GFR or glomerular filtration) )).mp.

4. 1 and 2 and 3.

5. Limit four to yr= '2009-Current'.

\section{Study records}

Using Covidence software, two independent reviewers will screen titles and abstracts. Any discrepancies will be resolved through discussion after completion of screening and third-party opinion will be sought. After attaining full texts of screened studies, eligible papers will be selected by two independent reviewers based on the inclusion/ exclusion criteria. Discrepancies will be resolved with discussion at the end of this stage and where necessary, third reviewer's opinion will be sought. Full text of two to five of the included studies will be selected randomly to evaluate the pilot data extraction questionnaire. Any further information that is not considered in the pilot form will be added after being approved by the review team. Title, abstract and full text of the included studies will then be carefully read by two independent reviewers to extract the required data according to a predefined questionnaire (online supplementary appendix 1). Any existing discrepancies in data extraction will be discussed and where necessary, a third reviewer will be involved. If the included studies were sufficiently homogenous, meta-analysis of the aggregated data will be conducted. Calibration exercises will be performed at each level of the process.

The results of the systematic review will be reported based on the Preferred Reporting Items for Systematic
Reviews and Meta-Analyses (PRISMA) statement, and the results will be presented following the PRISMA flow diagram (figure 1). ${ }^{27}$ The current systematic-review protocol has been written according to the PRISMA Protocols 2015 Statement. ${ }^{28}$

\section{Data items}

Using a data extraction form (online supplementary appendix 1), the following information from each study will be extracted:

1. The details of the included study with the first author as the study ID (the title, type of study, period of study, the year of publication, the country of origin).

2. The inclusion and exclusion criteria of the study.

3. Definition of diabetes.

4. Sample size (number of participants with type 1 or type 2 diabetes).

5. Demographic information (mean age, sex distribution, mean blood pressure, mean body mass index).

6 . Index test, its calculation and whether it was corrected for body surface area.

7. Creatinine measurement method, calibration coefficient, traceability to isotope dilution mass spectrometry.

8. Persons executing and interpreting index tests.

9. Reference test (exogenous filtration marker used to measure GFR), its clearance or plasma disappearance, whether it was a single injection or continuous infusion. If a single injection was done, whether the reported mGFR had Brochner-Mortensen correction.

10. Persons executing and interpreting reference test.

11. Setting of data collection.

12. Time interval between the index and reference test and whether any interventions were carried between.

13. Mean of the eGFR derived from creatinine-based CKD-EPI equation.

14. Mean of the mGFR value.

15. The definition of bias, precision and accuracy.

16. The reported bias, precision and accuracy.

17. Bland-Altman plot and the limit of agreement.

18. Scatter plot of the mGFR versus eGFR and line of perfect concordance.

19. Any report of Lin's concordance correlation, intra-class correlation coefficient or reduced major axis regression analysis.

20. Any reports on change in mGFR and eGFR in time.

21. Results based on diabetes type (type 1 and type 2).

22. Results based on GFR category (GFR $<60 \mathrm{~mL}$ / $\min / 1.73 \mathrm{~m}^{2}$, GFR $60-90 \mathrm{~mL} / \mathrm{min} / 1.73 \mathrm{~m}^{2}$ and GFR $\geq 90 \mathrm{~mL} / \mathrm{min} / 1.73 \mathrm{~m}^{2}$ ).

\section{Outcomes and prioritisation}

The primary outcome is to investigate bias, precision and accuracy of the creatinine-based CKD-EPI reported eGFR compared with the values measured by gold standard GFR measurement tools. If possible, the Bland-Altman graphs and $95 \%$ limits of agreement will be reviewed as well. As a secondary outcome, if possible, subgroup summarisation 


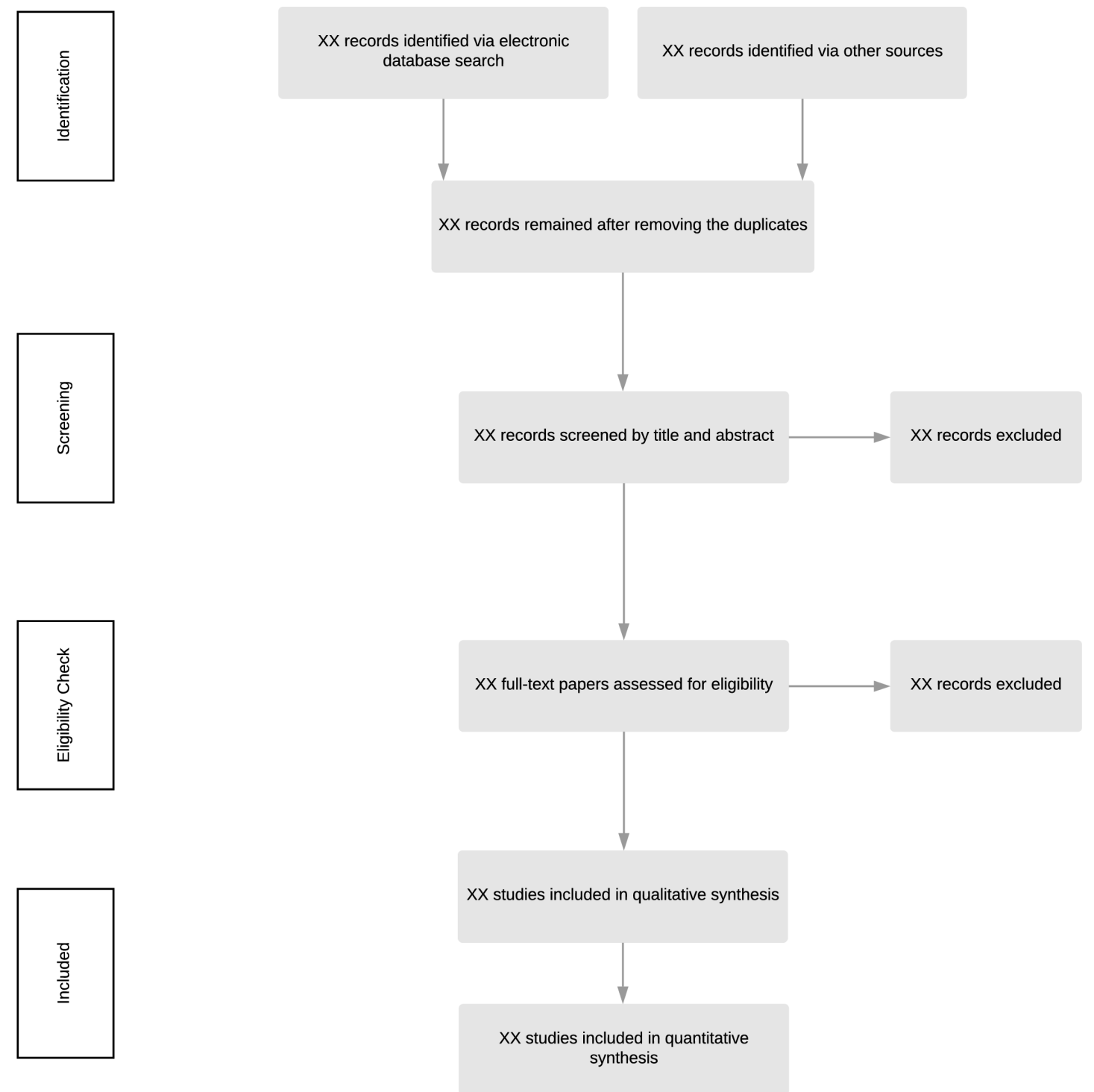

Figure 1 Preferred Reporting Items for Systematic Reviews and Meta-Analyses flow chart for reporting the process of selecting papers and number of papers in each phase.

and analysis of the diagnostic performance of the creatinine-based CKD-EPI equation based on participants diabetes type (type 1 and type two diabetes) and GFR category (GFR $<60 \mathrm{~mL} / \mathrm{min} / 1.73 \mathrm{~m}^{2}$, GFR $60-90 \mathrm{~mL}$ / $\min / 1.73 \mathrm{~m}^{2}$ and GFR $\geq 90 \mathrm{~mL} / \mathrm{min} / 1.73 \mathrm{~m}^{2}$ ) will be conducted.

\section{Risk of bias assessment}

The Quality Assessment of Diagnostic Accuracy Studies (QUADAS) $-2^{29}$ will be used to assess the design and methods of the included papers and assess the risk of bias as well as applicability. QUADAS-2 tool has four domains (ie, patient selection, index test, reference standard and flow and timing) to evaluate the quality of the methods and the reporting of studies that validated the performance of the index test in the target population. the tool completion process has four stages namely (1) report the review question, (2) develop review-specific guidance, (3) review the published flow diagram for the primary study or construct a flow diagram if none is reported and (4) judge bias and applicability. In all four domains, the risk of bias is assessed (low, high, unclear). Assessment of applicability (low, high, unclear) takes place only in the first three domains. Two reviewers will independently conduct the quality assessment. Any disagreement will be solved by discussion and where required, third party consultation will be sought. The presence of potential biases within the studies will be reported descriptively.

\section{Data analysis}

Heterogeneity will be judged visually by inspection of forest plots as well as using $\mathrm{I}^{2}$ statistics defined as ${ }^{3031}$ :

$I^{2}=100 \% \times\left(\chi^{2}\right.$ statistic-1 degree of freedom $) / \chi^{2}$ statistic

Summary of the proposed accuracy, precision and bias of the creatinine-base CKD-EPI eGFR as the index test compared with mGFR as the reference test will be reported. Bias will be defined as either mean or median of the difference between eGFR and mGFR (eGFR-mGFR). 
Precision will be defined as either $1 \mathrm{SD}$ or the IQR of the bias. Accuracy will be evaluated as the proportion of the eGFRs that are within a specific percentage (eg, 10\% $\left.\left(\mathrm{P}_{10}\right)\right)$ of the mGFRs. Where possible, the values will be calculated if not reported and 95\% CIs will be calculated when they are not reported in the articles. A bivariate random effect model will be used to derive summary estimates of precision, accuracy and bias, and their $95 \%$ CIs.

To equalise the participation of each study in the analysis, statistical weights based on the number of participants and measurements in each study will be calculated. In studies in which the number of measurements $(\mathrm{m})$, exceeded the number of participants (n), each measurement will receive the weight $\mathrm{n} / \mathrm{m}$. Measurements in studies with equal or fewer identified measurements than participants will receive the weight 1.

Any GFR expressed in millilitres per minute will be converted to millilitres per minute per $1.73 \mathrm{~m}^{2}$ assuming a body surface area of $1.73 \mathrm{~m}^{2}$. This conversion does not affect bias and accuracy measures expressed in per cent. However, the Bland-Altman diagrams will be affected to some extent.

Besides reporting the creatinine measurement method, calibration coefficient and traceability to isotope dilution mass spectrometry, we will conduct a subgroup analysis in studies which have clearly stated that the creatinine is measured by a method standardised to a mass spectroscopy creatinine standard.

Where possible, validation statistics will be aggregated and stratified based on diabetes type (type 1 and type 2 diabetes) and GFR category. GFR categories will be inspected as defined by the Kidney Disease: Improving Global Outcomes CKD definition that is: G1 $\left(\right.$ GFR $\geq 90 \mathrm{~mL} / \mathrm{min} / 1.73 \mathrm{~m}^{2}$ ), G2 (GFR $60-90 \mathrm{~mL}$ / $\left.\min / 1.73 \mathrm{~m}^{2}\right)$, G3a $\left(\right.$ GFR $\left.45-60 \mathrm{~mL} / \mathrm{min} / 1.73 \mathrm{~m}^{2}\right)$, G3b (GFR $30-45 \mathrm{~mL} / \mathrm{min} / 1.73 \mathrm{~m}^{2}$ ), G4 (GFR $15-30 \mathrm{~mL}$ / $\mathrm{min} / 1.73 \mathrm{~m}^{2}$ ) and G5 (GFR $<15 \mathrm{~mL} / \mathrm{min} / 1.73 \mathrm{~m}^{2}$ ). Alternatively, if this categorisation is not used by all the included studies, a broader categorisation defined as GFR $<60 \mathrm{~mL} / \mathrm{min} / 1.73 \mathrm{~m}^{2}$, GFR $60-90 \mathrm{~mL} / \mathrm{min} / 1.73 \mathrm{~m}^{2}$ and GFR $\geq 90 \mathrm{~mL} / \mathrm{min} / 1.73 \mathrm{~m}^{2}$ will be used.

If the quantitative synthesis of data is not possible, an aggregated summary of creatinine-base CKD-EPI derived eGFR, mGFR (specifying the exogenous marker used), demographic data as well as bias, accuracy and precision of the creatinine-base CKD-EPI eGFR as the index test compared with mGFR as the reference test will be reported.

\section{Meta-bias(es)}

To assess papers for selective outcome reporting bias, we will use the Outcome Reporting Bias in Trials classification system. ${ }^{32}$ To address the publication bias, research teams with available data on mGFR in people with diabetes will be contacted to check whether they had conducted studies with negative results which have not been published.

\section{Confidence in cumulative evidence}

The quality of evidence will be assessed using Grading of Recommendations Assessment, Development and Evaluation approach. ${ }^{33}$

\section{Patient and public involvement}

As this is a protocol for a systematic-review of the existing literature, reporting or planning for patients and public involvement is not applicable.

\section{ETHICS AND DISSEMINATION}

An outline of the protocol has been published in the PROSPERO International Prospective Register of Systematic Reviews in 2018. The results will summarise the studies that assessed the diagnostic performance of the creatinine-based CKD-EPI equation in people with diabetes. Where possible, a quantitative synthesis of the bias, precision and accuracy data will be provided and the outcomes using different algorithms will be discussed. Findings of the review will be presented at relevant scientific conferences and disseminated through publication in a peer-reviewed journal.

As a limitation, we will not be able to extract data from studies in other languages than English, Chinese, Farsi and Dutch.

\section{Author affiliations}

${ }^{1}$ Department of Medicine, The University of Melbourne, Austin Health, Melbourne, Victoria, Australia

${ }^{2}$ Florey Institute of Neuroscience and Mental Health, Heidelberg,Melbourne, Victoria, Australia

${ }^{3}$ Department of Endocrinology \& Diabetes, St Vincent's Hospital, The University of Melbourne, Fitzroy, Melbourne, Victoria, Australia

${ }^{4}$ Austin Health Sciences Library, Austin Health, Heidelberg, Victoria, Australia

Contributors NZ drafted the manuscript. All authors contributed to the development of the selection criteria, the risk of bias assessment strategy and data extraction criteria. HB and KK developed the search strategy. LC provided statistical expertise. EE, NT and RM provided expertise on diabetic kidney disease and glomerular filtration rate assessment. All authors read, provided feedback and approved the final manuscript.

Funding NZ was supported by the Melbourne Research Scholarship.

Competing interests None declared.

Ethics approval This review protocol will use publicly available data without directly involving human participants; hence, approval from an ethics committee is not required.

Provenance and peer review Not commissioned; externally peer reviewed.

Open access This is an open access article distributed in accordance with the Creative Commons Attribution Non Commercial (CC BY-NC 4.0) license, which permits others to distribute, remix, adapt, build upon this work non-commercially, and license their derivative works on different terms, provided the original work is properly cited, appropriate credit is given, any changes made indicated, and the use is non-commercial. See: http://creativecommons.org/licenses/by-nc/4.0/.

\section{REFERENCES}

1. GBD 2016 Disease and Injury Incidence and Prevalence Collaborators. Global, regional, and national incidence, prevalence, and years lived with disability for 328 diseases and injuries for 195 countries, 1990-2016: a systematic analysis for the global burden of disease study 2016. Lancet 2017;390:1211-59. 
2. Molitch ME, Adler Al, Flyvbjerg A, et al. Diabetic kidney disease: a clinical update from kidney disease: improving global outcomes. Kidney Int 2015;87:20-30.

3. de Boer IH, Rue TC, Hall YN, et al. Temporal trends in the prevalence of diabetic kidney disease in the United States. JAMA 2011;305.

4. Macisaac RJ, Ekinci El, Jerums G. Markers of and risk factors for the development and progression of diabetic kidney disease. Am J Kidney Dis 2014;63:39-62.

5. Matsushita K, van der Velde M, Astor BC, et al. Association of estimated glomerular filtration rate and albuminuria with all-cause and cardiovascular mortality in general population cohorts: a collaborative meta-analysis. Lancet 2010;375:2073-81.

6. Fox CS, Matsushita K, Woodward M, et al. Associations of kidney disease measures with mortality and end-stage renal disease in individuals with and without diabetes: a meta-analysis. Lancet 2012;380:1662-73.

7. Bjornstad P, Cherney DZ, Maahs DM. Update on estimation of kidney function in diabetic kidney disease. Curr Diab Rep 2015;15:57.

8. Gaspari F, Ruggenenti P, Porrini E, et al. The GFR and GFR decline cannot be accurately estimated in type 2 diabetics. Kidney Int 2013;84:164-73.

9. Maclsaac RJ, Ekinci El, Premaratne E, et al. The chronic kidney Disease-Epidemiology collaboration (CKD-EPI) equation does not improve the underestimation of glomerular filtration rate (GFR) in people with diabetes and preserved renal function. BMC Nephrol 2015;16:198.

10. Lewis EJ, Hunsicker LG, Bain RP, et al. The effect of angiotensinconverting-enzyme inhibition on diabetic nephropathy. The Collaborative Study Group. N Engl J Med 1993;329:1456-62.

11. Lewis EJ, Hunsicker LG, Clarke WR, et al. Renoprotective effect of the angiotensin-receptor antagonist irbesartan in patients with nephropathy due to type 2 diabetes. N Engl J Med 2001;345:851-60.

12. Brenner BM, Cooper ME, de Zeeuw D, et al. Effects of losartan on renal and cardiovascular outcomes in patients with type 2 diabetes and nephropathy. N Engl J Med 2001;345:861-9.

13. Levey AS, Eckardt K-U, Tsukamoto Y, et al. Definition and classification of chronic kidney disease: a position statement from kidney disease: improving global outcomes (KDIGO). Kidney Int 2005;67:2089-100.

14. Stevens LA, Coresh J, Greene T, et al. Assessing kidney function-measured and estimated glomerular filtration rate. $N$ Engl J Med 2006;354:2473-83.

15. Levey AS, Bosch JP, Lewis JB. A more accurate method to estimate glomerular filtration rate from serum creatinine: a new prediction equation. Ann Intern Med 1999;130:461.

16. Stevens LA, Coresh J, Feldman HI, et al. Evaluation of the modification of diet in renal disease study equation in a large diverse population. J Am Soc Nephrol 2007;18:2749-57.

17. Miller WG. Reporting estimated GFR: a laboratory perspective. Am Kidney Dis 2008;52:645-8.
18. Earley A, Miskulin D, Lamb EJ, et al. Estimating equations for glomerular filtration rate in the era of creatinine standardization. Ann Intern Med 2012;156:785-95.

19. Madero M, Sarnak MJ. Creatinine-based formulae for estimating glomerular filtration rate. Curr Opin Nephrol Hypertens 2011;20:622-30.

20. Rognant N, Lemoine S, Laville M, et al. Performance of the chronic kidney disease epidemiology collaboration equation to estimate glomerular filtration rate in diabetic patients. Diabetes Care 2011;34:1320-2.

21. Silveiro SP, Araújo GN, Ferreira MN, et al. Chronic kidney disease epidemiology collaboration (CKD-EPI) equation pronouncedly underestimates glomerular filtration rate in type 2 diabetes. Diabetes Care 2011;34:2353-5.

22. Maple-Brown LJ, Ekinci El, Hughes JT, et al. Performance of formulas for estimating glomerular filtration rate in Indigenous Australians with and without Type 2 diabetes: the eGFR Study. Diabet Med 2014;31:829-38.

23. Camargo EG, Soares AA, Detanico AB, et al. The chronic kidney disease epidemiology collaboration (CKD-EPI) equation is less accurate in patients with type 2 diabetes when compared with healthy individuals. Diabet Med 2011;28:90-5.

24. Zhao F, Zhang L, Lu J, et al. The chronic kidney disease epidemiology collaboration equation improves the detection of hyperfiltration in Chinese diabetic patients. Int J Clin Exp Med 2015;8:22084-97.

25. Stevens LA, Schmid CH, Zhang YL, et al. Development and validation of GFR-estimating equations using diabetes, transplant and weight. Nephrol Dial Transpl 2010;25:449-57.

26. Liu X, Qiu X, Shi C, et al. Modified glomerular filtration rateestimating equations developed in Asiatic population for Chinese patients with type 2 diabetes. Int J Endocrinol 2014;2014

27. Moher D, Liberati A, Tetzlaff J, et al. Preferred reporting items for systematic reviews and meta-analyses: the PRISMA statement. Ann Intern Med 2009;151:264.

28. Moher D, Shamseer L, Clarke M, et al. Preferred reporting items for systematic review and meta-analysis protocols (PRISMA-P) 2015 statement. Syst Rev 2015;4:1.

29. Whiting PF, Rutjes AWS, Westwood ME, et al. QUADAS-2: a revised tool for the quality assessment of diagnostic accuracy studies. Ann Intern Med 2011;155:529.

30. Higgins JPT, Thompson SG. Quantifying heterogeneity in a metaanalysis. Stat Med 2002;21:1539-58

31. Higgins JPT, Thompson SG, Deeks JJ, et al. Measuring inconsistency in meta-analyses. BMJ 2003;327:557-60.

32. Kirkham JJ, Dwan KM, Altman DG, et al. The impact of outcome reporting bias in randomised controlled trials on a cohort of systematic reviews. BMJ 2010;340:c365.

33. Guyatt G, Oxman AD, Akl EA, et al. Grade guidelines: 1. IntroductionGRADE evidence profiles and summary of findings tables. J Clin Epidemiol 2011;64:383-94. 
Correction: Diagnostic performance of the Chronic Kidney Disease Epidemiology Collaboration (CKD-EPI) equation at estimating glomerular filtration rate in adults with diabetes mellitus: a systematic review and meta-analysis protocol

Zafari N, Churilov L, MacIsaac RJ, et al. Diagnostic performance of the Chronic Kidney Disease Epidemiology Collaboration (CKD-EPI) equation at estimating glomerular filtration rate in adults with diabetes mellitus: a systematic review and meta-analysis protocol. BMJ Open 2019;9:e031558. doi: 10.1136/bmjopen-2019-031558

This article was previously published with incomplete author name.

The correct name for last author is Elif I Ekinci.

Open access This is an open access article distributed in accordance with the Creative Commons Attribution Non Commercial (CC BY-NC 4.0) license, which permits others to distribute, remix, adapt, build upon this work non-commercially, and license their derivative works on different terms, provided the original work is properly cited, appropriate credit is given, any changes made indicated, and the use is non-commercial. See: http://creativecommons.org/licenses/by-nc/4.0/.

(c) Author(s) (or their employer(s)) 2019. Re-use permitted under CC BY-NC. No commercial re-use. See rights and permissions. Published by BMJ.

BMJ Open 2019;9:e031558corr1. doi:10.1136/bmjopen-2019-031558corr1

Check for updates 